\title{
Enhanced Accuracy of Single-Molecule Diffusion Measurements with a Photocleavable Energy-Transfer Dyad**
}

\author{
Maren Dill, Moritz C. Baier, Stefan Mecking, and Dominik Wöll*
}

Single-molecule fluorescence spectroscopy and microscopy (SMFSM) are powerful techniques to investigate the structure and dynamics in biological and material science systems. ${ }^{[1-3]}$ One of the reasons for their success is the possibility to localize isolated molecules with a precision well beyond the diffraction limit of light ${ }^{[4,5]}$ and to connect their positions to trajectories. ${ }^{[6]}$ In this way, wide-field fluorescence microscopy allows the parallel detection of single-molecule motion. ${ }^{[7]}$ However, so far, absolute molecule positions have been used for analysis. ${ }^{[8]}$ Thus, the accuracy of single-molecule positioning and tracking is limited by the signal-to-noise ratio of their fluorescence signal, fluorescent background, ${ }^{[9]}$ molecule orientation, ${ }^{[10]}$ and the mechanical stability of the optical system during measurement. Furthermore, the determination of diffusion coefficients from single-molecule tracking strongly depends on experimental conditions ${ }^{[11]}$ and can introduce significant uncertainties. ${ }^{[8]}$ Even though nanometer accuracies have been reported under optimized experimental conditions, ${ }^{[12,13]}$ most systems of practical importance lack this precision owing to inherent background and insufficient longtime stability of the optical system. Several of the aforementioned problems are solved if positioning is performed versus an internal reference point, a concept which is exploited herein.

In this work, we present a functional fluorescence dye dyad with efficient energy transfer, which disappears after photocleavage and sufficient diffusional separation of both fluorophores. In particular, we report the synthesis, chemical and photophysical characterization, and application of a photocleavable energy-transfer dyad for single-molecule spectromicroscopy, and demonstrate the power of the dyad for two-color tracking and for accurate measurements of low diffusion coefficients $\left(D<10^{-19} \mathrm{~m}^{2} \mathrm{~s}^{-1}\right)$ using the internally referenced Förster distances instead of absolute positions.

Dyad 1 (Scheme 1) was synthesized as described in the Supporting Information. Intramolecular assemblies of pery-

[*] M. Dill, M. C. Baier, Prof. Dr. S. Mecking, Dr. D. Wöll

Fachbereich Chemie, Universität Konstanz

Universitätsstrasse 10, 78457 Konstanz (Germany)

E-mail: dominik.woell@uni-konstanz.de

Dr. D. Wöll

Zukunftskolleg, Universität Konstanz

Universitätsstrasse 10, 78457 Konstanz (Germany)

[***] This work was supported by the German research foundation (DFG, project WO 1670/2-1). D.W. thanks the Zukunftskolleg of the University of Konstanz for financial and administrative support. M.C.B. gratefully acknowledges the Fonds der chemischen Industrie for a scholarship. We are furthermore grateful to Prof. Dr. A.

Zumbusch and Prof. Dr. Ulrich E. Steiner for fruitful discussions.

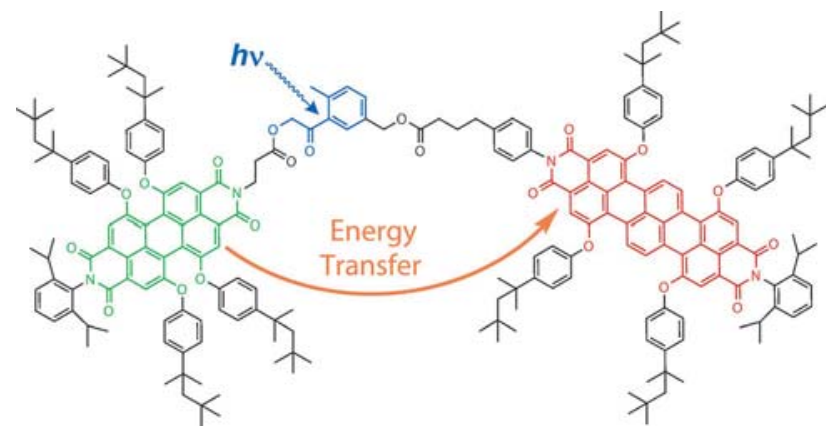

Scheme 1. Photocleavable fluorescence dye dyad 1 synthesized and used in this work. A perylene diimide chromophore serves as the energy donor, a terrylene diimide chromophore as the energy acceptor. Both moieties are covalently connected using a methyl phenacyl derivative as photolabile linker.

lene diimide (PDI) and terrylene diimide (TDI) derivatives ${ }^{[14]}$ have been described, ${ }^{[15-18]}$ but their linkage using a photolabile moiety is new. The absorption and emission spectra of the dyad $\mathbf{1}$ and the separated chromophores in cyclohexane are presented in the Supporting Information.

Depending on the excitation wavelength and thus the probabilities of the three different chromophores to absorb the excitation light, different photophysical and photochemical behavior can be observed in dyad 1. Excitation with long wavelengths above $600 \mathrm{~nm}$ exclusively excites the TDI chromophore and results in fluorescence with an intensity maximum at $699 \mathrm{~nm}$, bathochromically shifted by $18 \mathrm{~nm}$ compared to the emission maximum of dye $\mathbf{3}$ (structure and spectrum shown in the Supporting Information). Light of wavelengths shorter than $600 \mathrm{~nm}$ can be absorbed by both the PDI and the TDI moieties. However, owing to energy transfer, even after excitation of the PDI chromophore, the emission spectrum remains clearly dominated by the terrylene diimide emission. Assuming a FRET mechanism, the dependence of the energy-transfer efficiency $\eta$ on the distance between energy donor and energy acceptor ${ }^{[19]}$ is given by Equation (1):

$\eta\left(r, R_{0}\right)=\frac{1}{1+\left(\frac{r}{R_{0}}\right)^{6}}$

where $R_{0}$ is the Förster radius, which depends on the overlap between the donor emission and acceptor absorption spectrum and the orientation of both dyes; for our donor-acceptor pair it was determined to be $6.4 \mathrm{~nm}$ by assuming free rotation. Initially, energy transfer with $99 \%$ efficiency is observed owing to the close distance between both fluorophores. At such close distances, the dipolar approximation of Förster's 

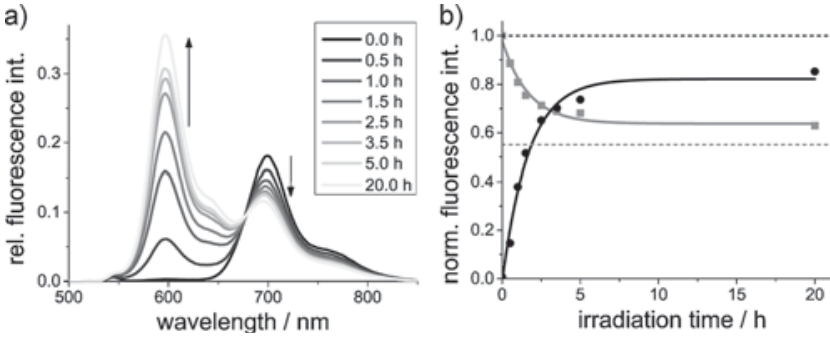

Figure 1. a) Time series of fluorescence spectra for irradiation of a homogeneous solution of dyad 1 in cyclohexane with $311 \mathrm{~nm}$. An area of $1 \mathrm{~cm}^{2}$ of a $3 \mathrm{~mL}$ standard cuvette was illuminated with an irradiation power of $5.2 \mathrm{~mW} \mathrm{~cm}^{-2}$. b) Evolution of the intensity of the fluorescence maxima of energy donor (circles) and energy acceptor (squares) with irradiation time. Both datasets were fitted with monoexponential functions with a time constant of $1.68 \mathrm{~h}$. The dashed lines represent the fluorescence intensities of dye $\mathbf{2}$ and dye $\mathbf{3}$ (structures: Supporting Information) at the same concentration, respectively.

theory is only a poor estimate owing to strong electronic coupling ${ }^{[20]}$ where exchange interactions have to be additionally considered. However, as shown in the Supporting Information, Section S8, our approach is rather robust as long as there is a significant drop of the energy-transfer rate at around $R_{0}$, a distance for which Förster's theory is valid and energy transfer proceeds by a FRET mechanism. ${ }^{[20]}$ UV light of wavelengths below $330 \mathrm{~nm}$ can be absorbed by all chromophores. In the case of absorption of a photon by the photolabile linker, it can be cleaved. The photokinetics of the cleavage reaction was investigated in homogeneous cyclohexane solution. After photocleavage, the energy donor and energy acceptor can diffuse independently and their average distance will increase continuously thus resulting in a gradual disappearance of the energy transfer (Figure 1a). Following the evolution of bulk fluorescence spectra and the time dependence of the donor and the acceptor fluorescence intensity for different exposure times to an irradiation wavelength of $311 \mathrm{~nm}$ (see Figure $1 \mathrm{~b}$ ), we determined a cleavage quantum yield of 0.03 after excitation of the photolabile phenacyl chromophore (see also Supporting Information), which is one order of magnitude smaller than the cleavage quantum yield of 0.22 for the photolabile moiety reported by Zabadal et al. ${ }^{[21]}$ Photophysical interactions of the excited photolabile moiety and the other chromophores are presumably responsible for this change in quantum yield, as is obvious from spectral shifts (Supporting Information, Figure S1).

Apart from the spectroscopic bulk measurements described above, the potential of dyad $\mathbf{1}$ for single-molecule microspectroscopy beyond what can be learnt with a single chromophore will be demonstrated. The essential advantage lies in the fact that prior to UV illumination both fluorophores are located in direct proximity (ca. $1 \mathrm{~nm}$ ). After photocleavage, both dyes can be positioned and tracked independently with a spatial resolution mainly determined by the signal-to-noise ratio ${ }^{[9]}$ of their emission.

To demonstrate this, we embedded dyad 1 into a circa $0.3 \mu \mathrm{m}$-thin poly(ethyl acrylate) (PEA) film $\left(M_{\mathrm{w}}=\right.$ $\left.100 \mathrm{~kg} \mathrm{~mol}^{-1}\right)$. The glass transition temperature $\left(T_{\mathrm{g}}\right)$ of PEA
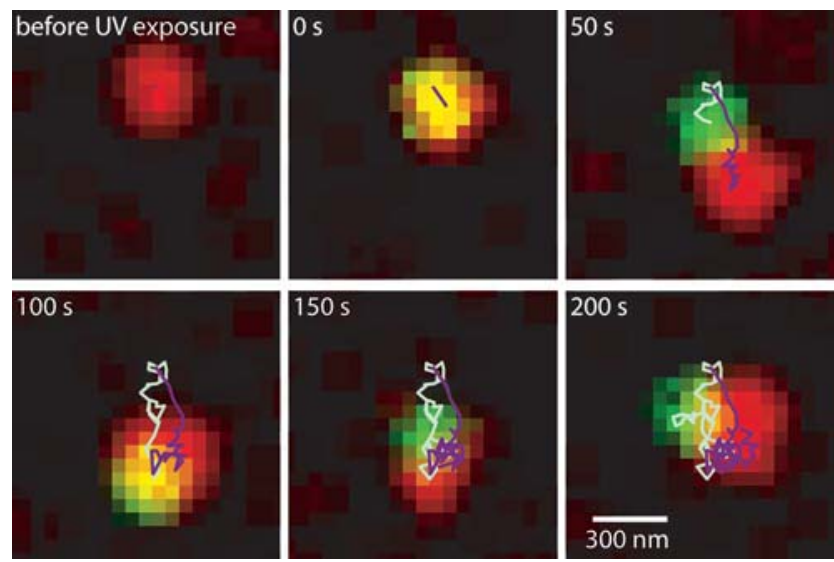

Figure 2. Series of false-color images taken from a widefield microscopy movie of dyad $\mathbf{1}$ in a PEA film at different times after a 5 s long UV irradiation (1 s integration time per image, see the Supporting Information). The green signal corresponds to the PDI channel, the red signal corresponds to the TDI channel. Yellow pixels: areas with intensity in both channels. The spots of both colors could be tracked independently (PDI moiety track: white; TDI moiety: violet).

was determined to be $-7.6^{\circ} \mathrm{C}$ using differential scanning calorimetry (DSC). In such a thin PEA film, the diffusion coefficient of dyad $\mathbf{1}$ is $7.2 \times 10^{-17} \mathrm{~m}^{2} \mathrm{~s}^{-1}$, a value high enough to yield a reasonably observable separation time after photocleavage of the dyad by UV irradiation.

Figure 2 shows a series of images taken at different times after UV irradiation for an initially single dyad $\mathbf{1}$ molecule. The PDI and TDI detection channels are overlapped in false colors. Before exposure to UV light, emission of the TDI chromophore of dyad $\mathbf{1}$ is observed owing to efficient energy transfer. After photocleavage of the dyad and diffusive separation of both chromophores to a distance above their Förster radius, PDI and TDI fluorescence can be detected in separate emission channels, and the position of the chromophores can be tracked independently with a spatial accuracy typical for single-molecule tracking ${ }^{[22]}$ without significant cross-talk between the channels.

Two-color single-molecule tracking works well for determining the time evolution of the distance between both cleaved moieties for distances above their positioning accuracy. Even though localization accuracies in the nanometer range have been reported, ${ }^{[12]}$ in several systems of practical importance the positioning accuracy is limited rather to about $10 \mathrm{~nm}$, which is due to inherent background signal or, in highly viscous media, preferred orientation of molecules. ${ }^{[10]}$ For these cases, we can use single-molecule energy-transfer efficiencies $^{[23-25]}$ to extend the spatial single-molecule-pair resolution.

To observe changes in energy-transfer efficiency on the single-molecule level in real-time, we embedded tiny amounts of dyad 1 molecules into a circa $0.3 \mu \mathrm{m}$-thin poly(butyl methacrylate) (PBMA) film $\left(M_{\mathrm{w}}=4 \mathrm{~kg} \mathrm{~mol}^{-1}\right)$ with a $T_{\mathrm{g}}$ of $16.2^{\circ} \mathrm{C}$. The time evolution of the fluorescence intensity after photocleavage is shown in Figure $3 \mathrm{a}-\mathrm{c}$ and was detected using cycling of the three different settings described as follows (see also the Supporting Information): 1) Laser excitation at $561 \mathrm{~nm}$, detection between 594 and $646 \mathrm{~nm}$ (PDI channel, 

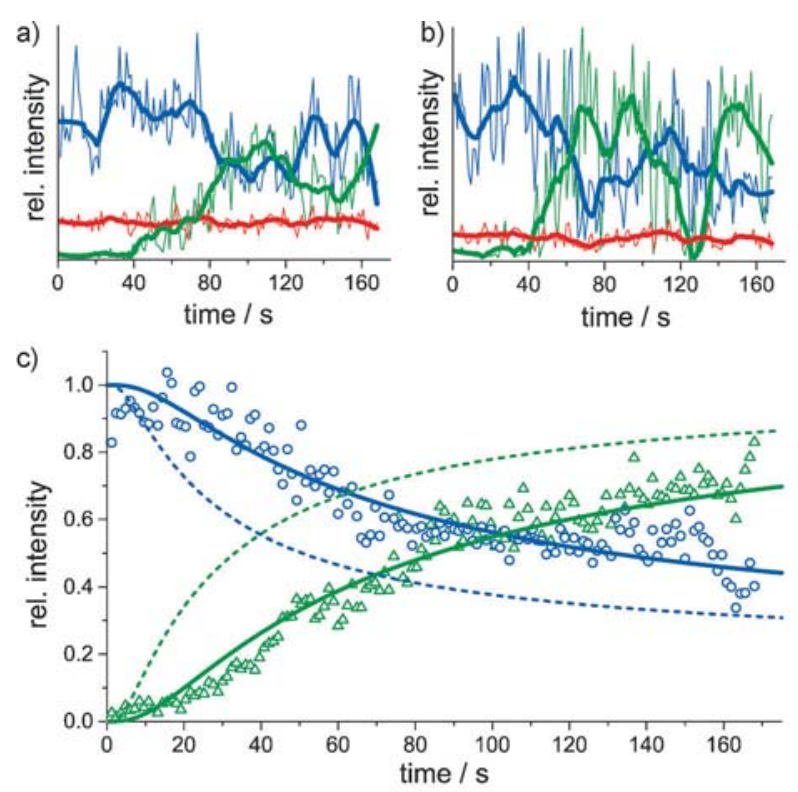

d)

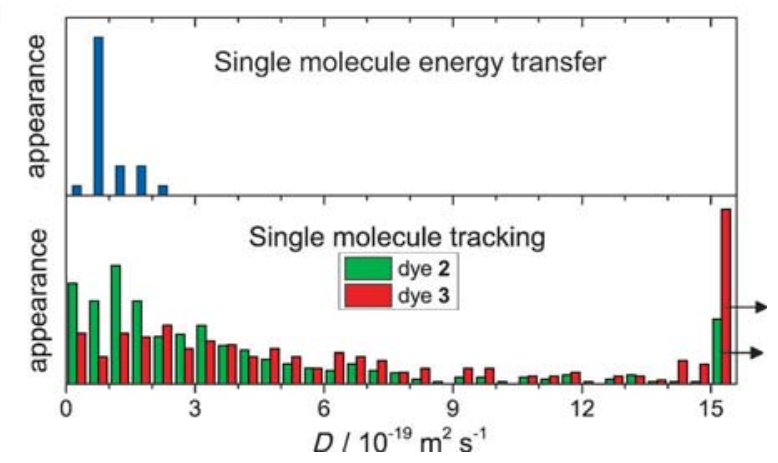

Figure 3. a, b) Typical time traces of single-molecule fluorescence after photocleavage of dyad 1 embedded in PBMA by UV light in the PDI channel (green), the TDI channel (red), and the FRET channel (blue). The moving averages over 10 points are shown as thick lines. c) Intensity traces averaged over 24 donors (green triangles) and acceptors (blue circles). Fits according to Equation (2)-(5) are presented as solid lines for a diffusion coefficient $D_{1,2} \approx 0.5 \times 10^{-19} \mathrm{~m}^{2} \mathrm{~s}^{-1}$, and as dashed lines for the sum of $3.1 \times 10^{-19} \mathrm{~m}^{2} \mathrm{~s}^{-1}$ for the diffusion coefficients of PDI dye $\mathbf{2}$ and TDI dye $\mathbf{3}$. d) Distributions of singlemolecule diffusion coefficients from single-molecule tracking of PDIdye $\mathbf{2}$ (green) and TDI-dye $\mathbf{3}$ (red), and from the fragments after photo cleavage (blue) using our approach with Equations (2)-(5). The analysis of a significant fraction of diffusion coefficients determined using single-molecule tracking resulted in values above $15 \times 10^{-19} \mathrm{~m}^{2} \mathrm{~s}^{-1}$, as indicated by the arrow.

green traces); 2) laser excitation at $658 \mathrm{~nm}$, detection between 672 and $712 \mathrm{~nm}$ (TDI channel, red traces); and 3) laser excitation at $561 \mathrm{~nm}$, detection between 672 and $712 \mathrm{~nm}$ (energy-transfer channel, blue traces). Figure $3 \mathrm{a}, \mathrm{b}$ show the intensity traces of two typical single molecules. Directly after UV irradiation (for $2 \mathrm{~s}$ ) and bond cleavage, both cleaved fragments are still in close proximity and thus efficient energy transfer is observed. Gradually, the probability that their distance separation exceeds the Förster radius, and thus the intensity in the energy-transfer channel decreases and the intensity in the PDI channel increases significantly. Owing to the dependence of the FRET efficiency on the inverse of the sixth power of distance, FRET changes are sensitive to distance variation, in particular at distances close to the Förster distance. Data shown in Figure $3 \mathrm{a}, \mathrm{b}$ reveal that this point is reached at about $60-80 \mathrm{~s}$. As diffusion of the dye fragments in our polymer film is a statistical process, the distances between fragments of the dyad after photocleavage can fluctuate as shown in Figure $3 \mathrm{~b}$.

In the following, we compare the diffusion coefficients obtained from our energy-transfer approach and singlemolecule tracking. We use a model in which both fluorophores are initially situated at the same position, after photocleavage separate by normal diffusion, and energy transfer proceeds by a FRET mechanism. On the basis of this assumption, the probability $P$ of finding two fluorophores with a diffusion coefficient of $D_{1}$ and $D_{2}$, respectively, at a distance $r$ after a certain time $t$ can be expressed as Equation (2) with $D=D_{1}+D_{2}:{ }^{[26]}$

$P(r, t, D)=\frac{1}{6 \pi D t} \exp \left(-\frac{r^{2}}{6 D t}\right) 2 \pi r$

This results in a FRET efficiency $\eta$ given by Equation (3):

$\eta(t, D)=\int_{r=0}^{\infty} P(r, t, D) \eta\left(r, R_{0}\right) \mathrm{d} r$

Normalized donor and acceptor intensities $I$ are calculated by Equation (4) and (5):

$I_{\mathrm{D}}=1-\eta(t, D)$

$I_{\mathrm{A}}=0.8 \eta(t, D)+0.2$

Note that the acceptor efficiency is corrected to account for the excitation of a TDI molecule with the green $561 \mathrm{~nm}$ laser. Equations (2)-(5) allow for an estimation of the diffusion coefficients of a single molecule with an accuracy limited by diffusion statistics.

Least-square fitting of the curves from Equations (4) and (5) to the intensities of 24 photocleaved dyad 1 molecules (see the Supporting Information) resulted in a distribution of diffusion coefficients as shown in Figure $3 \mathrm{~d}$ centered around a value of $D_{1,2} \approx 0.5 \times 10^{-19} \mathrm{~m}^{2} \mathrm{~s}^{-1}$ assuming that both cleaved moieties have the same diffusion coefficient owing to their similar size. This value is in good agreement with the one obtained by fitting the summed intensities of the 24 intensity traces (Figure $3 \mathrm{c}$ ).

In contrast, the diffusion coefficient obtained for PDI-dye 2 and TDI-dye 3 (structures see Supporting Information) using single-molecule tracking are significantly higher $(1.3 \times$ $10^{-19} \mathrm{~m}^{2} \mathrm{~s}^{-1}$ and $1.8 \times 10^{-19} \mathrm{~m}^{2} \mathrm{~s}^{-1}$, respectively). As shown in Figure $3 \mathrm{c}$ (dashed lines), these diffusion coefficients do not match with our energy-transfer measurements. The distribution of single-molecule diffusion coefficients (Figure $3 \mathrm{~d}$ ) obtained by single-molecule tracking is very broad and reaches from about $50 \times 10^{-19} \mathrm{~m}^{2} \mathrm{~s}^{-1}$ to even negative values, which are due to the statistical inaccuracies of the meansquare displacement analysis. The breadth of this distribution of diffusion coefficients could be easily misinterpreted as heterogeneity in molecular motion. 
A comparison with our energy-transfer approach, however, shows clearly that the diffusion coefficients are distributed around $0.5 \times 10^{-19} \mathrm{~m}^{2} \mathrm{~s}^{-1}$ with a standard deviation of $\pm 0.3 \times 10^{-19} \mathrm{~m}^{2} \mathrm{~s}^{-1}$, and thus under the applied conditions motion in the polymer is still rather homogeneous. It should also be emphasized that it was necessary to measure for more than 100 min to detect sufficient motion for reliable singlemolecule tracking, whereas the energy-transfer measurements only took 5 minutes.

The reason for the higher accuracy of our FRET method to determine low diffusion coefficients lies in several advantages of this method over single-molecule tracking. These advantages are demonstrated in the most straightforward way

using the mean-square displacement $D_{\lim } \propto \frac{\left\langle x_{\lim }^{2}\right\rangle}{t}$.

First, the sensitivity length scale $x_{\lim }$ of the spatial accuracy is determined solely by the Förster radius and does not depend on localization accuracy, that is, it is not limited by the signal-to-noise ratio of spots and background as long as sufficient intensity from the energy donor and the energy acceptor can be detected. Furthermore, positioning errors owing to instabilities in the optical system do not increase $x_{\lim }$ and thus $D_{\text {lim }}$. As energy-transfer efficiency depends only on the relative distance between donor and acceptor but not on their absolute coordinates, it is not sensitive to drifts of the optical system as long as the single dyads and their fragments can be uniquely assigned to each other from frame to frame. The fact that the signal-to-noise ratio of spots does not significantly limit the accuracy of our approach is another advantage over single-molecule tracking in that lower excitation intensities can be used, thus decreasing the probability of early photobleaching of the dyes. The lower excitation intensities and the insensitivity against optical destabilization allows for significantly increased observation times $t$ which further result in a lower value of $D_{\text {lim }}$. It should be also noted that the energy-transfer method is sensitive to $3 \mathrm{D}$ motion, whereas most single-molecule tracking approaches detect a $2 \mathrm{D}$ projection of $3 \mathrm{D}$ motion, which for isotropic diffusion is accounted for by using a $4 D t$ relation for the mean square displacement instead of $6 D t$.

For FRET, the efficiency also depends on the relative orientation of energy donor and energy acceptor, as expressed by the so called orientation factor $\kappa$. Therefore, we simulated the effect of reorientation of the cleaved moieties on the energy-transfer efficiency (see Supporting Information). It could be shown that, in the investigated system, rotational motion can cause noticeable fluctuations in the intensity time trace, but not to an extent as to corrupt the overall determination of $D$.

In conclusion, we developed and photochemically characterized a photocleavable energy-transfer dyad and demonstrated its applicability for single-molecule microspectroscopy. After photocleavage, it allows for following the evolution of distances between both fragments from the nm to the $\mu \mathrm{m}$ length scale using single-molecule energy-transfer efficiencies and independent two-color tracking, respectively. Observation of changes in the single-molecule energy-transfer efficiencies between both fragments enables the determi- nation of low single-molecule diffusion coefficients and their distribution with high accuracy compared to single-molecule tracking. Future work will focus on exploiting our novel approach to measure slow diffusion in polymer systems and to extend it to other fluorophores and photolabile moieties.

Keywords: diffusion - fluorescence microscopy . Förster resonance energy transfer - photolabile groups . single-molecule spectroscopy

[1] F. Kulzer, T. Xia, M. Orrit, Angew. Chem. 2010, 122, 866-879; Angew. Chem. Int. Ed. 2010, 49, 854-866.

[2] D. Wöll, E. Braeken, A. Deres, F. De Schryver, H. Uji-i, J. Hofkens, Chem. Soc. Rev. 2009, 38, 313-328.

[3] P. Tinnefeld, M. Sauer, Angew. Chem. 2005, 117, 2698-2728; Angew. Chem. Int. Ed. 2005, 44, 2642-2671.

[4] B. Huang, M. Bates, X. W. Zhuang in Annu. Rev. Biochem., Vol. 78, Annual Reviews, Palo Alto, 2009, S. 993-1016.

[5] A. M. van Oijen, J. Köhler, J. Schmidt, M. Müller, G. J. Brakenhoff, Chem. Phys. Lett. 1998, 292, 183-187.

[6] M. J. Saxton, Nat. Methods 2008, 5, 671-672.

[7] T. Schmidt, G. J. Schütz, W. Baumgartner, H. J. Gruber, H. Schindler, Proc. Natl. Acad. Sci. USA 1996, 93, 2926-2929.

[8] D. Ernst, J. Köhler, Phys. Chem. Chem. Phys. 2013, 15, 845-849.

[9] K. I. Mortensen, L. S. Churchman, J. A. Spudich, H. Flyvbjerg, Nat. Methods 2010, 7, 377-383.

[10] J. Enderlein, E. Toprak, P. R. Selvin, Opt. Express 2006, 14, $8111-8120$

[11] X. Michalet, A. J. Berglund, Phys. Rev. E 2012, 85, 061916.

[12] A. Yildiz, P. R. Selvin, Acc. Chem. Res. 2005, 38, 574-582.

[13] A. Yildiz, J. N. Forkey, S. A. McKinney, T. Ha, Y. E. Goldman, P. R. Selvin, Science 2003, 300, 2061-2065.

[14] T. Weil, T. Vosch, J. Hofkens, K. Peneva, K. Müllen, Angew. Chem. 2010, 122, 9252-9278; Angew. Chem. Int. Ed. 2010, 49, $9068-9093$.

[15] E. Fron, L. Puhl, I. Oesterling, C. Li, K. Müllen, F. C. De Schryver, J. Hofkens, T. Vosch, ChemPhysChem 2011, 12, 595-608.

[16] G. Hinze, R. Métivier, F. Nolde, K. Müllen, T. Basché, J. Chem. Phys. 2008, 128, 124516.

[17] R. Métivier, F. Nolde, K. Müllen, T. Basché, Phys. Rev. Lett. 2007, 98, 047802 .

[18] C. Curutchet, B. Mennucci, G. D. Scholes, D. Beljonne, J. Phys. Chem. B 2008, 112, 3759-3766.

[19] J. R. Lakowicz, Principles of Fluorescence Spectroscopy, 2nd ed., Kluwer Academic/Plenum Publishers, 1999.

[20] S. E. Braslavsky, E. Fron, H. B. Rodriguez, E. S. Roman, G. D. Scholes, G. Schweitzer, B. Valeur, J. Wirz, Photochem. Photobiol. Sci. 2008, 7, 1444-1448.

[21] M. Zabadal, A. P. Pelliccioli, P. Klán, J. Wirz, J. Phys. Chem. A 2001, 105, 10329-10333.

[22] D. Wöll, C. Kölbl, B. Stempfle, A. Karrenbauer, Phys. Chem. Chem. Phys. 2013, 15, 6196-6205.

[23] R. Roy, S. Hohng, T. Ha, Nat. Methods 2008, 5, 507-516.

[24] B. Schuler, W. A. Eaton, Curr. Opin. Struct. Biol. 2008, 18, $16-$ 26.

[25] T. Ha, T. Enderle, D. F. Ogletree, D. S. Chemla, P. R. Selvin, S. Weiss, Proc. Natl. Acad. Sci. USA 1996, 93, 6264-6268.

[26] M. J. Saxton, Biophys. J. 1997, 72, 1744-1753. 